Tropical Journal of Pharmaceutical Research October 2020; 19 (10): 2171-2177

ISSN: $1596-5996$ (print); 1596-9827 (electronic) (C) Pharmacotherapy Group, Faculty of Pharmacy, University of Benin, Benin City, 300001 Nigeria.

\title{
A cross-sectional assessment of health-related quality of life among healthcare providers in Alkharj, Saudi Arabia
}

\author{
Ahmed A Albassam, Muhammad Shahid lqbal*, Mohammad Ruhal Ain, Anwar \\ Alotaibi, Abdullah Althemery, Abdullah Alfaifi \\ Department of Clinical Pharmacy, College of Pharmacy, Prince Sattam bin Abdulaziz University, Al-Kharj 11942, Saudi Arabia
}

*For correspondence: Email: m.javed@psau.edu.sa; Tel: +966 115886057

Sent for review: 28 May 2020

Revised accepted: 20 September 2020

\begin{abstract}
Purpose: To determine the current level of health-related quality of life (HRQoL), and evaluate factors influencing it among health-care providers (HCPs) in Alkharj, Saudi Arabia.

Methods: A prospective cross-sectional study was conducted among HCPs in Alkharj, Saudi Arabia, using the standardized research tool, BREF-WHOQOL. A self-administered questionnaire was distributed in hospitals, in the medical colleges of Prince Sattam Bin Abdulaziz University, and community pharmacies in Alkharj, Saudi Arabia. Descriptive, comparative, and inferential statistics were performed using SPSS.

Results: A total of 289 HCPs agreed to participate in the study, $52 \%$ of whom were female. Most participants were married (69 \%), and most had fewer than 10 years of experience (58\%). Statistically significant differences in marital status, education level, income, and year of practice were found that correlated with HCPs' HRQoL ( $p \leq 0.05)$. Overall, respondents reported an above-average score for all survey items.

Conclusion: The results indicate that HCPs see their general quality of life as good - a particularly important consideration in view of the demanding nature of their job. This study will help the concerned authorities to exactly know the current level of HRQoL among HCPs in Alkharj, Saudi Arabia, as well as help in designing better healthcare policies in Saudi Arabia.
\end{abstract}

Keywords: Healthcare providers (HCP), Health-related quality of life (HRQoL), Healthcare policies, Occupational stress

\begin{abstract}
This is an Open Access article that uses a fund-ing model which does not charge readers or their institutions for access and distributed under the terms of the Creative Commons Attribution License (http://creativecommons.org/licenses/by/4.0) and the Budapest Open Access Initiative (http://www.budapestopenaccessinitiative.org/read), which permit unrestricted use, distribution, and reproduction in any medium, provided the original work is properly credited.

Tropical Journal of Pharmaceutical Research is indexed by Science Citation Index (SciSearch), Scopus, International Pharmaceutical Abstract, Chemical Abstracts, Embase, Index Copernicus, EBSCO, African Index Medicus, JournalSeek, Journal Citation Reports/Science Edition, Directory of Open Access Journals (DOAJ), African Journal Online, Bioline International, Open-J-Gate and Pharmacy Abstracts
\end{abstract}

\section{INTRODUCTION}

The health status of health-care providers (HCPs) is important and can affect their performance [1]. Quality of life is a multidimensional aspect that can be used to assess the general wellbeing of individuals and societies [2]. Health-related quality of life (HRQoL) represents the influences of health state, health policies, and medical action on perceptions of welfare [3]. Studies show that poor health status among HCPs is associated with a decrease in the quality and safety of practices. Moreover, undesirable outcomes such as medication errors increase [1-3]. The decrease in physical and/or mental HRQoL among HPCs is attributed to 
many factors, for example, workload and shifts, years of work, number of patients, poor patient outcomes, disagreement with a peer, poor management, and lack of support $[1,4]$.

A number of studies have shown that the HRQoL of health-care providers is low. A study in a Taiwanese hospital found that $47 \%$ of the staff had either minor psychiatric or depressive disorder, especially among nurses and pharmacists [2-5]. In Greece, nurses reported a high level of job dissatisfaction, and their health status was reportedly average [6]. An HRQoL study among Spanish health-care providers found a low HRQoL compared to the reference population, especially in the mental components [7]. In Jeddah, Saudi Arabia, approximately $60 \%$ of the nurses working at King Abdul-Aziz University Hospital were satisfied with their general health [8].

A variety of methods for estimating the quality of life are available to health-care providers, for example, the World Health Organization Quality of Life Instrument WHOQOL[9]. The WHOQOL measures domains pertaining to health, relationships, and environments and enables researchers to cross-compare cultures [9]. Skevington and colleagues (2018) detected a positive relationship between the survey's domains and sustainable development goals, which promote wellbeing to all individuals. Colby and colleagues (2018) found an association between quality of life, measured through the WHO survey, and the medical students' work burnout [10]. However, most of these studies were limited to physicians and medical students rather than all health-care providers.

Identifying HRQoL and occupational stressors among HPCs in Saudi Arabia is significant in supporting their health. Any improvement in HRQoL among HCPs will directly reflect patient care and health outcomes. Studies evaluating HRQoL and job satisfaction among HCPs in Saudi Arabia are limited. Therefore, this study was sought to evaluate the quality of life among HCPs and to explore the relationship between work-related stresses, job satisfaction, and general health of HCPs.

\section{EXPERIMENTAL}

\section{Sampling and study setting}

A cross-sectional study was conducted to determine the current level of HRQoL, and evaluate various factors influencing HRQoL, among HCPs in Alkharj, Saudi Arabia. A selfadministered

questionnaire was distributed in local hospitals. Participants were also recruited from colleges of medicine, pharmacy, and applied medical sciences, as well as from 38 community pharmacies. Convenience sampling was used to select research subjects who met the study inclusion criteria. The study's population included physicians, dentists, pharmacists, nurses, and other health-care providers practicing in hospitals, clinics, or the medical community or working in academia. All participants had at least a bachelor's degree in a medical specialty; potential participants who did not meet the inclusion criteria were excluded from the study.

\section{Instrument}

A questionnaire was used to collect information. Participants' demographic data gender, age, nationality, marital status, professional degree, nature of the job, job title, highest educational level, years of practice, income, and smoking was collected. A standardized research tool, the BREF-WHOQOL [9], was used to measure the quality of life (QOL). The BREF-WHOQOL consists of 26 items that have four domains reflecting QOL: physical health, psychological, social, and environmental. The first domain, the physical health, contains questions related to sleep and resting, fatigue and energy, mobility, discomfort and pain, medical treatment during daily life, and satisfaction with their capability for work. The second domain, the psychological, concentrates on the capability to focus, selfconfidence, body image, spirituality, and the frequency of positive or negative moods.

The third domain, the social relationships, consists of questions connected to satisfaction with relationships at a personal level, social assistance, and satisfaction with sexual life. A fourth domain, the environmental, contains questions related to safety, satisfaction with the home and their physical environment, financial fulfillment, accessibility of both health and social care, information and leisure activity, and satisfaction with transportation. Furthermore, overall QOL and general health are captured. Each domain produces a score; the sum of the total scores ranges from 0 and 100, with a high score indicates a better QOL, according to WHOQOL guidelines [9]. The score helps to evaluate people's perception regarding culture and values and their personal objectives and involvements.

\section{Ethical permission}

All procedures performed in this study complied with the ethical standards of Prince Sattam Bin 
Abdulaziz University's Institutional Review Board (IRB) and received research committee approval (no. PSAU/COM/RC/IRB/A/13), and complied with the guidelines of 1964 Helsinki Declaration and its amendments [11]. The study began after receiving approval from the Prince Sattam Bin Abdulaziz University IRB. All participants signed an informed consent form before starting the questionnaire, and all the collected forms were kept strictly confidential and only used for the research. Before data collection, authorization was obtained from the WHO to utilize the WHOQOL-BREF survey.

\section{Data analysis}

Descriptive statistics were used to evaluate demographics and the participants' social and specific characteristics. Frequencies with percentages and means with standard deviations were calculated for categorical and continuous variables, respectively. Cronbach's alpha for the four domains of WHOQOL-BREF was determined. To confirm the normality distribution of the data, the Shapiro-Wilk test and $Q-Q$ plots were used. An independent samples t-test and Pearson's correlation coefficient was used to evaluate correlations (agreements) between demographics and domains and to identify the differences in overall HRQoL and its domains. Data from the questionnaire were analyzed using Statistical Package for the Social Sciences (SPSS) version 22.0.

\section{RESULTS}

The demographic characteristics of the study's participants are presented in Table 1 . There were a total of 289 participants, with more females than males $(n=153,52.9 \%$ and $n=136,47.1 \%$ respectively). Two hundred thirty-five (81.3\%) participants were Saudi nationals, whereas 54 (18.7 \%) were Non-Saudis. Sixty-seven (23.2 \%) had a post-master's level of education and 222 (76.8\%) had a bachelor's or master's level education. One hundred sixty-seven (57.8\%) participants had attended continuous professional development (CPDs) or continuous medical education (CMEs) short courses, and 122 did not.

Table 2 depicts the participants' responses against each item of the questionnaire. The Cronbach's alpha for the entire WHOQOL-BREF was 0.915. The Cronbach's alpha for the physical, psychological, social and environmental domains exceeded 0.7 . Table 3 presents the mean HRQoL scores for all four domains of WHOQOL-BREF among the participants. The mean score for the physical health domain was $67.01 \pm$ 13.67. Mean scores for the psychological, social relationships, and environmental domains were $71.27 \pm 14.15$, $71.92 \pm 17.06$, and $68.00 \pm 14.73$, respectively.

Table 1: Demographic Characteristics of the Study Participants $(n=289)$

\begin{tabular}{lcc}
\hline Description & Frequency & $\%$ \\
\hline Gender & 136 & 47.1 \\
Male & 153 & 52.9 \\
$\begin{array}{l}\text { Female } \\
\text { Nationality }\end{array}$ & 54 & 18.7 \\
$\begin{array}{l}\text { Saudi } \\
\text { Non-Saudi }\end{array}$ & 235 & 81.3 \\
$\begin{array}{l}\text { Marital status } \\
\text { Single/separated/ }\end{array}$ & 89 & 30.8 \\
married & 200 & 69.2 \\
$\begin{array}{l}\text { Highest education } \\
\text { Doctorate/Residency }\end{array}$ & 67 & 23.2 \\
Bachelor/Master & 222 & 76.8 \\
$\begin{array}{l}\text { Experience } \\
\text { <10 years }\end{array}$ & 169 & 58.5 \\
>10 years & 120 & 41.5 \\
$\begin{array}{l}\text { Income } \\
\text { S4000 USD }\end{array}$ & 237 & 82.0 \\
$>4000$ USD & 52 & 18.0 \\
CPDs/CMEs & 167 & 57.8 \\
Yes & 122 & 42.2 \\
No & & \\
\hline
\end{tabular}

1 USD $=3.75$ SAR; CPD = Continuing Professional Development; $\mathrm{CME}=$ Continuing Medical Education

Table 4 shows the correlations between the four different domains of WHOQOL-BREF. Based on the observed findings, statistically significant positive correlations were noted between all four domains of WHOQOL-BREF. There was also a statistically significant positive correlation between the first two WHOQOL-BREF questions, that is, QoL and overall health satisfaction status, and scores obtained from different domains. The strength of correlation among QoL and overall health status was moderately-strong (Pearson's r $>0.4$ ), whereas four domains showed lowmoderate, strongly-moderate, and strong correlations (Pearson's $r$ ranged from 0.214 to 0.694).

Table 5 represents correlation coefficients and the bivariate relationship between demographic variables and the mean domain scores. Statistically significant differences were observed between the scores for marital status and the social and environmental domain $(p=0.02$ and 0.002 , respectively). Married participants had higher scores than singles. Respondents had significantly higher HRQoL scores in the social domain $(73.47 \pm 16.64)$ than in the environmental domain $(69.80 \pm 14.47)$. 
Table 2: Distribution of WHOQOL-BREF Items' Responses (frequency)

\begin{tabular}{lccccc}
\hline & WHOQOL-BREF items & & & \\
\hline Overall QoL \& Health & Very Poor & Poor & Average & Good & Very Good \\
\hline QoL Rating & 1 & 6 & 25 & 162 & 95 \\
Health Satisfaction Status & 4 & 10 & 35 & 179 & 61 \\
Get around Ability & 2 & 29 & 81 & 139 & 38 \\
Sleep Satisfaction & 9 & 28 & 90 & 135 & 27 \\
Body Appearance & 9 & 9 & 77 & 112 & 82 \\
Negative Feelings & 8 & 25 & 89 & 132 & 35 \\
Relationship Satisfaction & 1 & 8 & 49 & 154 & 77 \\
Sexual Satisfaction & 19 & 15 & 57 & 135 & 63 \\
Life Safety & 1 & 3 & 99 & 131 & 55 \\
Healthy Environment & 4 & 19 & 101 & 131 & 34 \\
Financial Satisfaction & 2 & 18 & 94 & 122 & 53 \\
Leisure Activities & 9 & 55 & 115 & 93 & 17 \\
Healthcare Satisfaction & 3 & 19 & 78 & 142 & 47 \\
Transport Satisfaction & 13 & 23 & 65 & 145 & 43 \\
\hline
\end{tabular}

Table 3: Mean HRQoL Scores for Four Domains of WHOQOL-BREF

\begin{tabular}{lc}
\hline Domain & $\begin{array}{c}\text { HRQoL scores } \\
\text { (mean士SD) }\end{array}$ \\
\hline Physical domain & $67.01 \pm 13.67$ \\
Psychological Domain & $71.27 \pm 14.15$ \\
Social Relationship & $71.92 \pm 17.06$ \\
Domain & $68.00 \pm 14.73$ \\
Environment Domain & \\
\hline
\end{tabular}

Statistically significant differences were observed between the scores for physical domain and environmental domain against highest education level ( $p=0.022$ and 0.006 , respectively) whereby the highly educated participants achieved the better scores than the other group. Statistically significant differences were also observed for physical and environmental domains against experience and income levels. Participants who attended CPDs or CMEs showed significantly higher HRQoL scores for the physical and psychological domains. Correlations between demographics and the WHOQOL-BREF domains are shown in Table 6. Statistically significant positive and negative correlations were observed between various demographic variables and the WHOQOL-BREF domains (Pearson's " $r$ " ranged from -0.166 to 0.206 ).

\section{DISCUSSION}

This study confirmed that HCPs in Saudi Arabia are moderately satisfied with their HRQoL in social, psychological and environment domains and are relatively less satisfied in their physical domain. Overall, the HCPs have better access to good health-care facilities, self-esteem, and social circles. This study also verified that married HCPs were more satisfied in their social and environmental domains than the singles. It was observed that highly educated HCPs had a better understanding of their own and their patients' disease states, which obviously helped them chose precise treatment regimens. In the past decade, HRQoL has been an emergent concept and an important treatment outcome parameter in assessing individuals' general health state and monitoring treatment efficacy and overall disease management [2-4]. This study determined HRQoL in the four different domains of WHOQOL-BREF among HCPs. These days, the severity of the chronic diseases demand that HCPs pay due attention to their own HRQoL and that of their patients. Researchers from across the globe have explored different aspects of HRQoL among HCPs, there are a limited number of literature exploring this phenomenon from Saudi Arabia.

Table 4: Correlation coefficients in overall health and domains of WHOQOL-BREF

\begin{tabular}{|c|c|c|c|c|c|c|}
\hline Factor ${ }^{*}$ & QoL & Overall & Physical & Psychological & Social & Environmenta \\
\hline QoL & 1 & & & & & \\
\hline Overall & 0.570 & 1 & & & & \\
\hline Physical & 0.398 & 0.390 & 1 & & & \\
\hline Psychological & 0.482 & 0.521 & 0.641 & 1 & & \\
\hline Social & 0.253 & 0.214 & 0.464 & 0.548 & 1 & \\
\hline Environmental & 0.371 & 0.331 & 0.694 & 0.625 & 0.54 & 1 \\
\hline
\end{tabular}

${ }^{*}$ All the correlation coefficients were significant at $p<0.001$ 
Table 5: Comparison of WHOQOL-BREF mean scores based on sociodemographic factors

\begin{tabular}{|c|c|c|c|c|c|c|}
\hline Variable & QoL & Overall & Physical & Psychological & Social & Environmental \\
\hline \multicolumn{7}{|l|}{ Gender } \\
\hline Male & 4.12 & 4.00 & 67.37 & 70.72 & 71.35 & 69.29 \\
\hline Female & 4.24 & 3.96 & 66.69 & 71.77 & 72.42 & 66.86 \\
\hline \multicolumn{7}{|l|}{ Nationality } \\
\hline Saudi & 4.22 & 3.79 & 65.62 & 70.01 & 71.55 & 69.57 \\
\hline Non-Saudi & 4.18 & 4.02 & 67.33 & 71.56 & 72.00 & 67.64 \\
\hline \multicolumn{7}{|l|}{ Marital status } \\
\hline Single/Separated & 4.23 & 4.07 & 65.10 & 71.39 & $68.43^{*}$ & $63.97^{* *}$ \\
\hline Married & 4.17 & 3.93 & 67.87 & 71.22 & $73.47^{*}$ & $69.80^{* *}$ \\
\hline \multicolumn{7}{|l|}{ Highest education } \\
\hline Doctorate/residency & 4.14 & 3.94 & $70.35^{*}$ & 72.38 & 74.14 & $72.34^{* *}$ \\
\hline Bachelor/master & 4.20 & 3.99 & $66.00^{*}$ & 70.94 & 71.24 & $66.69^{* *}$ \\
\hline \multicolumn{7}{|l|}{ Experience } \\
\hline$\leq 10$ years & 4.19 & 3.98 & $65.17^{\star *}$ & 70.66 & 70.65 & $65.69^{* * *}$ \\
\hline$>10$ years & 4.18 & 3.96 & $69.61^{* *}$ & 72.13 & 73.70 & $71.26^{* * *}$ \\
\hline \multicolumn{7}{|l|}{ Income } \\
\hline$\leq 4000$ USD & 4.18 & 3.98 & $66.05^{* \star}$ & 70.64 & 71.13 & $66.59^{* * *}$ \\
\hline$\overline{>} 4000$ USD & 4.21 & 3.94 & $71.38^{* *}$ & 74.15 & 75.48 & $74.46^{* * *}$ \\
\hline \multicolumn{7}{|l|}{ CPDs/CMEs ${ }^{a}$} \\
\hline Yes & 4.22 & 4.05 & $68.83^{* \star}$ & $73.28^{* *}$ & 73.38 & $70.04^{* *}$ \\
\hline No & 4.13 & 3.87 & $64.53^{* *}$ & $68.53^{* *}$ & 69.91 & $65.22^{\star *}$ \\
\hline
\end{tabular}

Table 6: Correlation between demographic variables and different domains of WHOQOL-BREF

\begin{tabular}{lcccccc}
\hline Variable & QoL & Overall & Physical & Psychological & Social & Environment \\
\hline Gender & .088 & -.025 & -.025 & .037 & .031 & -.082 \\
Race & -.022 & .114 & .049 & .043 & .010 & -.051 \\
Marital Status & -.043 & -.086 & .094 & -.005 & $.136^{*}$ & $.183^{* *}$ \\
Education & .032 & .028 & $-.134^{*}$ & -.043 & -.072 & $-.162^{* *}$ \\
Experience & -.008 & -.014 & $.160^{* *}$ & .051 & .088 & $.187^{* * *}$ \\
Income & .014 & -.022 & $.150^{*}$ & .095 & .098 & $.206^{* * *}$ \\
CPDs/CMEs ${ }^{a}$ & -.062 & -.113 & $-.156^{* *}$ & $-.166^{* *}$ & -.100 & $-.162^{* *}$ \\
${ }^{*} p<0.05,{ }^{* *} p<0.01,{ }^{* * *} p<0.001 ;{ }^{a}$ continuous professional development (CPDs) or continuous medical education \\
$($ CMEs)
\end{tabular}

Frequent access to medication use, annoyance, a variety of treatment regimens, and positive and negative psychological impacts are among the major concerns affecting HRQoL among HCPs $[12,13]$. In the current study, among the four domains of WHOQOL-BREF, the highest mean score (satisfaction level) was found in the social domain, possibly due to good health-care facilities, body appearance, no negative feelings, more positive feelings, a greater level of selfesteem, highly religious, spiritual applicability, personal beliefs, better memory, less dependence on self-pocket expenses, and acquiring better health-care needs [14].

Moreover, the lowest mean score (satisfaction level) was observed for the physical domain (67.01 \pm 13.67), indicating compromised activities of daily living, more dependence on medical treatment and health assistant, less mobility and more discomfort, fatigue, and less work capacity. Conversely, good mean scores were observed for the psychological and environment domains (71.27 \pm 14.15 and $68.00 \pm$
14.73, respectively), showing good individual relationships, greater social support and satisfactory sexual activities, good financial means, openings for new knowledge and skills, freedom in religious activities, better safety and security, free access to quality health care, and frequent access to cheap and convenient transportation [11-14]. This study found that income level has an important impact on individuals HRQoL. Those earning more than 4,000 USD (15,000 SAR) scored much higher in almost every domain of WHOQOL-BREF than the other groups. These differences may be due to better economic and social conditions with better access to financial resources, the quality of their health care, and better access to opportunities to acquire the latest medical information. Excellent lifestyle and high earnings significantly improved HRQoL scores in the main domains; physical, psychological, social, and environmental. Another contributing factor may be their social circle: Spending more time than with families and friends may positively affect their personal and social relationships. 
Undeniably, better income appears to be a unique predictor of improved HRQoL [12-15]. According to the findings of another study, income was significantly associated with the psychological and environmental domains and overall general health satisfaction states [15].

Higher education level often advances selfinterest and involvement in improving general health states, which is a key determinant of selfsatisfaction. It is also a common observation that highly educated HCPs have a better understanding of dosages, treatment regimens, and disease management [16]. In addition to pharmacotherapy, they are more likely to acclimatize their lifestyle and adopt preventive measures, resulting in improvement in overall HRQoL [17]. In both developed and developing countries across the globe, more educated individuals are reported to live longer and enjoy better health conditions and status compared to the less educated [14,17].In our study, highly educated HCPs have a better understanding of their disease states, which helps them better comply when taking medications $[13,18]$. This finding might be the reason why highly educated HCPs scored better in the HRQoL assessment.

There was a significant difference between married and single HCPs in the social and environmental domains, with higher scores associated with married HCPs. This might be because married life could play a vital role in maintaining good HRQoL, especially at an older age. Mutual understanding, moral support and assistance in tackling various family issues between married couples may positively influence their HRQoL [19]. This result validated previous concerns reported by Malik et al, who found that single health-care professionals experience higher stress levels [20]. This finding is particularly important because HCPs have demanding careers, and job control is significantly correlated with social relationships. Health-care managers can pay particular attention to these factors. Improving the work environment by, for example, promoting and presenting healthy activities and understanding complex social settings, may positively improve work conditions [21]. This study used the WHOQOL-BREF to measure domains pertaining to health, relationships, and environments. The results of this study support previous findings that the social aspect of HRQOL show the highest scores while physical health is the lowest among HCPs [22]. Promoting healthy activities and offering CPDs or CMEs inside the healthcare facility might provide an important protective factor for HRQOL among HCPs. This study suggests that improvements, especially in income level predictors, might lead to considerable progress in improving HRQoL among HCPs.

\section{Limitations of the study}

The WHOQOL-BREF is also a self-reported research instrument which may present some biasedness in data reporting. Another study limitation noted could be the cross-sectional design of the study. The third limitation could be lack of a control group to make applicable comparisons for the findings obtained. Despite some limitations, the current study reports overall HRQoL among HCPs in Alkharj, Saudi Arabia.

\section{CONCLUSION}

The findings from this study confirm that the WHOQOL-BREF research tool is a reliable instrument to measure HRQoL among HCPs in Saudi Arabia. From the obtained data it is evident that HCPs in Saudi Arabia enjoy overall good HRQoL though in some of the variables they showed relatively moderate or lower HRQoL scores. These lower or the negative HRQoL scores are may be due to diverse education levels, non-attendance of the CMEs, increased living costs, inability to work and less availability or enjoyment of social activities. The finding of this study is an imperative contribution in literature for the understanding of the overall HRQoL among HCPs in Saudi Arabia.

\section{DECLARATIONS}

\section{Acknowledgement}

This project was supported by the Deanship of Scientific Research at Prince Sattam bin Abdulaziz University through the research proposal No 2017/03/8275.

\section{Conflict of interest}

The authors declare that they have no conflict of interest with regard to this study.

\section{Contribution of authors}

We declare that this work was done by Dr. Albassam, Dr. Iqbal, Ruhal Ain, Alotaibi, Dr. Althemery, and Dr. Alfaifi and all liabilities pertaining to claims relating to the content of this article will be borne by the authors. Dr. Albassam contributed to study design, conception, IRB approval, data collection, and manuscript writing. Dr. Iqbal worked on data collection, analysis, and manuscript writing. Ruhal Ain took part in the 
analysis and data collection. Alotaibi collected part of the data. Dr. Althemery obtained the grant, data analysis and revision. Dr. Alfaifi Manuscript writing, revision, and editing.

\section{Open Access}

This is an Open Access article that uses a funding model which does not charge readers or their institutions for access and distributed under the terms of the Creative Commons Attribution License (http://creativecommons.org/licenses/by/ 4.0) and the Budapest Open Access Initiative (http://www.budapestopenaccessinitiative.org/rea d), which permit unrestricted use, distribution, and reproduction in any medium, provided the original work is properly credited.

\section{REFERENCES}

1. Fronteira I, Ferrinho $P$. Do nurses have a different physical health profile? A systematic review of experimental and observational studies on nurses' physical health. J Clin Nurs 2011; 20(17-18): 24042424.

2. Gregory $D$, Johnston $R$, Pratt $G$, Watts $M$, Whatmore S. The dictionary of human geography, John Wiley \& Sons, 2011.

3. Ebrahim S. Clinical and public health perspectives and applications of health-related quality of life measurement. Soc Sci Med 1995; 41(10): 1383-1394.

4. Shields $M$, Wilkins K. Factors related to on-the-job abuse of nurses by patients. Heal Reports 2009; 20(2): 7-19.

5. Leary S, Francis C. Healthy staff will enhance outcomes. Health Serv J 2007; 117(6056): 26-7.

6. Ioannou $P$, Katsikavali V, Galanis $P$, Velonakis $E$, Papadatou D, Sourtzi $P$. Impact of job satisfaction on Greek nurses' health-related quality of life. Saf Health Work 2015; 6(4): 324-328.

7. Suñer-Soler R, Grau-Martín A, Font-Mayolas S, Gras ME, Bertran C, Sullman MJM. Burnout and quality of life among Spanish healthcare personnel. J Psychiatr Ment Health Nurs 2013; 20(4): 305-313.

8. Ibrahim NK, Alzahrani NA, Batwie AA, Abushal RA, Almogati G, Sattam A, Hussin BK. Quality of life, job satisfaction and their related factors among nurses working in King Abdulaziz university hospital, Jeddah, Saudi Arabia. Contemp Nurse 2016; 52(4): 486-498.

9. World Health Organization. WHOQOL-BREF: Introduction, Administration, Scoring and Generic Version of the Assessment: Field Trial Version, December 1996.

10. Colby L, Mareka M, Pillay S, Sallie F, van Staden $C$, du Plessis E, Joubert $G$. The association between the levels of burnout and quality of life among fourth-year medical students at the university of the Free State. S Afr J Psychiatr 2018; 24: 1101.

11. World Medical Association Declaration of Helsinki: Ethical Principles for Medical Research Involving Human Subjects Adopted by the 18th WMA General Assembly, Helsinki, Finland, June, 1964, and amended by the 48th WMA General Assembly, Somerset West, Republic of South Africa, October, 1996. Accessed online 07-102019.

12. Joshi U, Subedi R, Poudel P, Ghimire PR, Panta S, Sigdel MR. Assessment of quality of life in patients undergoing hemodialysis using WHOQOL-BREF questionnaire: A multicenter study. Int J Nephrol Renovasc Dis 2017; 10: 195-203.

13. Elbur Al, Albarraq AA, Maugrabi MM, Alharthi SA. Knowledge of, satisfaction with and adherence to oral anticoagulant drugs among patients in King Fasial Hospital: Taif, Kingdom Saudi Arabia. Int J Pharm Sci Rev Res 2015; 31(1): 274-280.

14. Barcellona D, Contu P, Sorano GG, Pengo V, Marongiu $F$. The management of oral anticoagulant therapy: the patient's point of view. Thromb Haemost 2000; 83(1): 49-53.

15. Gemmell LA, Terhorst $L$, Jhamb $M$, Unruh $M$, Myaskovsky L, Kester L. Gender and racial differences in stress, coping, and health-related quality of life in chronic kidney disease. J Pain Symptom Manage 2016; 52(6): 806-812.

16. Ogutmen B, Yildirim A, Sever MS, Bozfakioglu S, Ataman $R$, Erek E, Cetin O, Emel A. Health-related quality of life after kidney transplantation in comparison intermittent hemodialysis, peritoneal dialysis, and normal controls. Transplant Proc 2006; 38(2): 419-21.

17. Bayoumi M, Al Harbi A, Al Suwaida A, Al Wakeel J, Mishkiry A. Predictors of quality of life in hemodialysis patients. Saudi J Kidney Dis Transpl 2013; 24(2): 254 9.

18. Daher AM, Ibrahim HS, Daher TM, Anbori AK. Health related quality of life among Iraqi immigrants settled in Malaysia. BMC Public Health 2011; 11: 407.

19. Song $T$, Ding $Y W$, Sun $Y, H e Y N, Q i D J, W u Y, W u B$, Lang L, Yu K, Zhao $X$ et al. A population-based study on health-related quality of life among urban community residents in Shenyang, Northeast of China. BMC Public Health 2015; 15: 921.

20. Malik M. Occupational stress among practicing pharmacists in Pakistan: the current dilemma. $J$ of Pharmacol \& Clin Res 2017; 4(2): 555633.

21. Dellve L, Eriksson A. Health-promoting managerial work: A theoretical framework for a leadership program that supports knowledge and capability to craft sustainable work practices in daily practice and during organizational change. Societies 2017; 7(2): 12.

22. Yang SC, Kuo PW, Wang JD, Lin MI, Su S. Quality of life and its determinants of hemodialysis patients in Taiwan measured with WHOQOL-BREF(TW). Am J Kidney Dis 2005; 46: 635-641.

Trop J Pharm Res, October 2020; 19(10): 2177 